\title{
Sex with a transgender or gender diverse person among patients attending a sexual health centre in Melbourne, Australia
}

\author{
Eoin F Cleere, ${ }^{1,2}$ Christopher K Fairley, ${ }^{1,3}$ Launcelot McGrath, ${ }^{1,2}$ \\ Catriona S Bradshaw, ${ }^{1,3}$ Marcus Y Chen, ${ }^{1,3}$ Eric P F Chow ${ }^{1,3}$
}

\begin{abstract}
- Additional material is published online only. To view please visit the journal online (http://dx.doi.org/10.1136/ sextrans-2018-053653).

'Melbourne Sexual Health Centre, Alfred Health, Melbourne, Victoria, Australia ${ }^{2}$ School of Medicine, Royal College of Surgeons in Ireland, Dublin, Ireland

${ }^{3}$ Central Clinical School, Monash University, Melbourne, Victoria, Australia
\end{abstract}

Correspondence to Dr. Eric P F Chow, Melbourne Sexual Health Centre, Carlton, VIC 3053, Australia; echow@ mshc.org.au

Received 15 April 2018 Revised 29 May 2018 Accepted 6 June 2018 Published Online First 27 June 2018
Check for updates

To cite: Cleere $\mathrm{EF}$, Fairley CK, McGrath L, et al. Sex Transm Infect 2019;95:46-52.

\begin{abstract}
Objectives All males and females attending the Melbourne Sexual Health Centre (MSHC) from August 2017 were asked whether they had had sex with a transgender or gender diverse (TGD) person using computer-assisted self-interviewing (CASI). We aimed to verify the self-reported responses via chart review. The secondary aim of this study was to identify whether having sex with a TGD person was associated with STI risk.
\end{abstract}

Methods This was a retrospective chart analysis of patients visiting MSHC between August and December 2017. Chart review was performed to verify the selfreported responses. Multivariable logistic regression was performed to examine the association between having sex with a TGD person and patients' characteristics and STI risk.

Results of the 10100 male and female consultations, the proportion who reported having sex with a TGD person was $111(1.0 \%)$ and was higher among males $(1.3 \%)$ than females $(0.6 \%)(p=0.001)$. After chart review, we could verify $66.9 \%$ of the responses, more for males $(75.2 \%)$ than females $(45.2 \%)(p<0.001)$. Of the 6822 males, men aged $\geq 35$ years (adjusted $\mathrm{OR}=2.2$; $95 \% \mathrm{Cl} 1.1$ to 4.1 ) were more likely to have sex with a TGD person compared with men aged $\leq 24$ years, after adjusting for confounding factors. Sex with a TGD person was not associated with sexual orientation in males. Of the 3278 females, gay and bisexual females had 13.7fold ( $95 \% \mathrm{Cl} 5.1$ to 37.0$)$ higher odds of having sex with a TGD person than heterosexual females. There was no association between chlamydia positivity and sex with a TGD person in both males and females.

Conclusion When a question on TGD partners is asked as part of routine sexual history using CASI, the majority of responses could be verified. TGD partners were most commonly reported among males. These findings underscore the value of asking patients about sex with TGD partners.

\section{INTRODUCTION}

Conventionally, sex and gender have been defined with the binary model as either male or female. Both social science and biomedical research have provided evidence that the binary model of sex definition is too simplistic, and a variety of categories is required. ${ }^{12}$ However, the redefinition is always a challenge due to the political, cultural and societal resistance. Transgender and gender diverse (TGD) people are an example that do not fit under the binary model; they are a group of people whose gender at birth differs from their gender identity. The lack of research on transgender individuals suggested the lack of awareness and understanding about their needs and health. ${ }^{34}$ A previous systematic review conducted in 2013 has estimated that the global HIV prevalence among transgender women was $19.1 \%$, but this prevalence varies considerably across geographical regions. ${ }^{5}$ This estimate may not be generalisable to the whole TGD population due to possible sampling bias such as small sample size and variations of recruitment settings. ${ }^{6}$ One would presume that because of this, it is likely that the risk of acquiring HIV or STIs might be different in someone who has sex with a TGD person. Australian clinicians did not identify a question regarding transgender partners as a core clinical question that should be asked of all individuals attending a sexual health service. ${ }^{7}$ Furthermore, the 2013 British Association for Sexual Health and HIV (BASHH) guidelines also do not indicate that patients should be routinely asked whether they have had transgender partners. ${ }^{8}$ To date, we are not aware of any data on the routine use of a question relating to whether 'sex with a TGD person' in sexual health services. However, given that the risk of HIV/STI is different in TGD persons, such a question could potentially identify individuals at increased risk of HIV/STI.

In response to client feedback about the absence of a question on sex with transgender individuals, we added such a question in our clinic using computer-assisted self-interviewing (CASI) in mid-August 2017 as part of our routine clinical care. All male and female patients who attend the Melbourne Sexual Health Centre (MSHC) were required to complete this question via CASI before they saw a clinician. At MSHC, patients can self-identify their sex as male, female, transgender, unknown or other. However, only patients who self-identified themselves as either male or female are required to complete CASI. We were unsure however if patients attending MSHC would understand the term 'transgender and gender diverse'. A previous study has found that less than $50 \%$ of medical students were able to define and understand the term 'transgender'. 9 Therefore, we were 
concerned how the broader patient population would respond to this question. The primary aim of this study was to determine the proportion of male and female patients self-reporting sex with a TGD person. In addition, we also wanted to explore how many of those self-reporting sex with a TGD person could have this response verified in their clinical record. The secondary aim of this study was to examine the association between having sex with a TGD person and patients' characteristics including risk of STIs.

\section{METHODS}

This study was a data analysis from a retrospectively collected clinical data at MSHC. The MSHC is the largest sexual health clinic in Victoria, Australia. It provides a free walk-in service for everyone who attends with around 48000 consultations in 2017. Patients attending MSHC for their first visit or those who have not attended MSHC in the previous 3 months are required to complete a detailed sexual history using CASI, which is available in English, Thai, Chinese and Korean. This is then collected and recorded electronically in the Clinical Practice Management System, which also records all patient demographic data, sexual behaviours and clinician notes during the consultation.
On the $16^{\text {th }}$ of August 2017, the following question was added to CASI for all male and female patients: 'In the past 12 months, have you had sex with a trans or gender diverse person?' Patients had the option to answer 'Yes', 'No', 'Unsure' or 'Decline to answer'. Electronic patients' records between the $16^{\text {th }}$ of August and $29^{\text {th }}$ of December 2017 were extracted. Patients were considered eligible in this analysis if they: (1) were male or female; (2) had attended MSHC within the study period; and (3) had answered the new question on sex with a TGD. Patients who identified as 'TGD', 'Unknown' or 'Other' were excluded as they were not asked this question via CASI. Patients who had only attended express consults such as the Test-And-Go (TAG) service or Nurse Express Testing Service (NETS) were also excluded because details about sexual history were not asked and documented during the consultation. ${ }^{10} 11$

A chart review was carried out on patients who answered 'Yes', 'Unsure' or 'Decline to answer' to the question on TGD partners via CASI to verify their self-reported responses. Patients who had their charts reviewed were assigned a verified response based on the electronic free-text medical history written by the attendant clinician stating if they had or had not had sex with a TGD person within the past 12 months. Those who had evidence recorded in

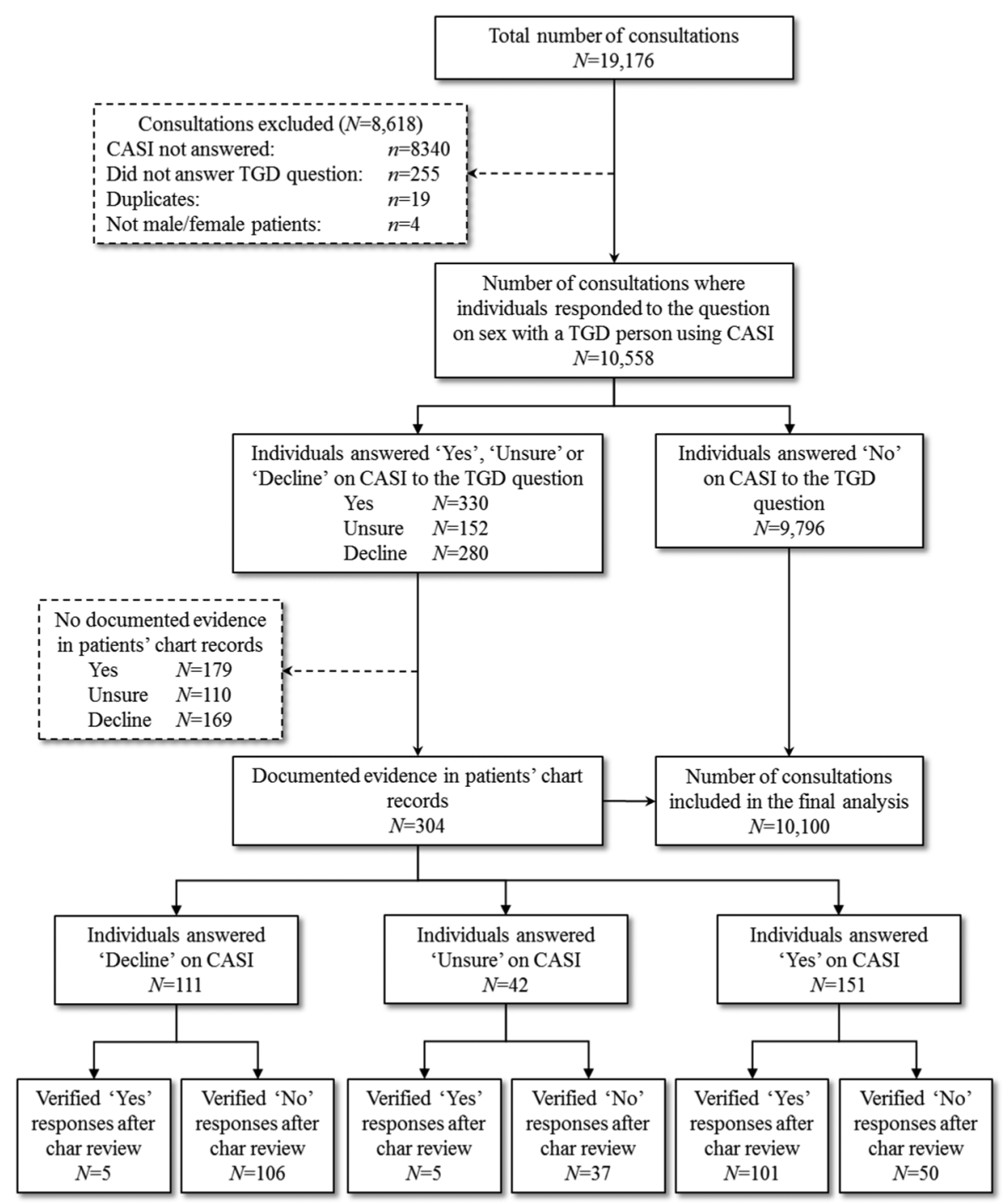

Figure 1 Flow diagram of sample selection. CASI, computer-assisted self-interviewing; TGD, transgender and gender diverse. 
Table 1 Verified responses on sex with a TGD person after chart review among 151 individuals who self-reported having sex with a TGD person using CASI

\begin{tabular}{|c|c|c|c|c|}
\hline & $\begin{array}{l}\text { Self-reported responses on sex with } \\
\text { a TGD person using CASI*, } n\end{array}$ & $\begin{array}{l}\text { Verified responses on sex } \\
\text { with a TGD person after } \\
\text { chart review, } n\end{array}$ & $\begin{array}{l}\text { Verified responses rate on } \\
\text { sex with a TGD person after } \\
\text { chart review, } \%\end{array}$ & $P$ valuest \\
\hline Gender $(n=151)$ & & & & $<0.001$ \\
\hline Male & 109 & 82 & 75.2 & \\
\hline Female & 42 & 19 & 45.2 & \\
\hline \multicolumn{5}{|l|}{ Males $(n=109)$} \\
\hline Age, years & & & & 0.804 \\
\hline$\leq 24$ & 16 & 11 & 68.8 & \\
\hline $25-34$ & 56 & 43 & 76.8 & \\
\hline$\geq 35$ & 37 & 28 & 75.7 & \\
\hline Country of birth $\ddagger$ & & & & 0.375 \\
\hline Australia & 58 & 41 & 70.7 & \\
\hline Overseas & 42 & 33 & 78.6 & \\
\hline Language spoken at home & & & & 0.306 \\
\hline English & 88 & 66 & 75.0 & \\
\hline Not English & 13 & 8 & 61.5 & \\
\hline Injecting drug use $\ddagger$ & & & & 0.189 \\
\hline Yes & 5 & 5 & 100.0 & \\
\hline No & 100 & 74 & 74.0 & \\
\hline Sexual orientation & & & & 0.785 \\
\hline Gay or bisexual & 71 & 54 & 76.1 & \\
\hline Heterosexual & 38 & 28 & 73.7 & \\
\hline Sex workers $\ddagger$ & & & & 0.569 \\
\hline Yes & 1 & 1 & 100.0 & \\
\hline No & 106 & 80 & 75.5 & \\
\hline Number of partners§ & & & & 0.873 \\
\hline$\leq 3$ & 27 & 20 & 74.1 & \\
\hline$>3$ & 82 & 62 & 75.6 & \\
\hline \multicolumn{5}{|l|}{ Females $(n=42)$} \\
\hline Age, years & & & & 0.562 \\
\hline$\leq 24$ & 17 & 6 & 35.3 & \\
\hline $25-34$ & 19 & 10 & 52.6 & \\
\hline$\geq 35$ & 6 & 3 & 50.0 & \\
\hline Country of birth & & & & 0.474 \\
\hline Australia & 24 & 12 & 50.0 & \\
\hline Overseas & 18 & 7 & 38.9 & \\
\hline Language spoken at home $\ddagger$ & & & & 0.227 \\
\hline English & 37 & 18 & 48.6 & \\
\hline Not English & 5 & 1 & 20.0 & \\
\hline Injecting drug use & & & & 0.439 \\
\hline Yes & 3 & 2 & 66.7 & \\
\hline No & 39 & 17 & 43.6 & \\
\hline Sexual orientation & & & & 0.429 \\
\hline Lesbian or bisexual & 26 & 13 & 50.0 & \\
\hline Heterosexual & 16 & 6 & 37.5 & \\
\hline Sex workers $\ddagger$ & & & & 0.006 \\
\hline Yes & 11 & 9 & 81.8 & \\
\hline No & 30 & 10 & 33.3 & \\
\hline Number of partners§ & & & & 0.273 \\
\hline$\leq 3$ & 14 & 8 & 57.1 & \\
\hline$>3$ & 28 & 11 & 39.3 & \\
\hline
\end{tabular}

*Individuals who answered 'Yes' on CASI to the question on having sex with a TGD person.

tP values were calculated from $\chi^{2}$ test.

$\ddagger$ Total values in these categories do not match total number due to missing data.

§Of any gender within the past 12 months.

CASI, computer-assisted self-interviewing; MSM, men who have sex with men; TGD, transgender and gender diverse; WSW, women who have sex with women. 
Table 2 Factors associated with having sex with a TGD person among 6822 males attending the Melbourne Sexual Health Centre

\begin{tabular}{|c|c|c|c|c|c|c|}
\hline Factors & $\begin{array}{l}\text { Number of individuals, } \\
\mathrm{n}\end{array}$ & $\begin{array}{l}\text { Number of individuals who } \\
\text { had had sex with a TGD } \\
\text { person, } n(\%)\end{array}$ & $\begin{array}{l}\text { Crude OR } \\
(95 \% \mathrm{Cl})\end{array}$ & $P$ values & $\begin{array}{l}\text { Adjusted OR } \\
(95 \% \mathrm{Cl})\end{array}$ & $P$ values \\
\hline \multicolumn{7}{|l|}{ Age, years } \\
\hline $25-34$ & 3222 & $46(1.4)$ & 1.80 (0.97 to 3.34$)$ & 0.063 & $1.78(0.96$ to 3.30$)$ & 0.068 \\
\hline$\geq 35$ & 1973 & $33(1.7)$ & 2.11 (1.11 to 4.03$)$ & 0.023 & 2.17 (1.14 to 4.14$)$ & 0.019 \\
\hline \multicolumn{7}{|l|}{ Country of birth* } \\
\hline \multicolumn{7}{|l|}{ Injecting drug use* } \\
\hline No & 6552 & $86(1.3)$ & 1 & Ref & & \\
\hline Yes & 117 & $2(1.7)$ & $1.31(0.32$ to 5.38$)$ & 0.710 & & \\
\hline \multicolumn{7}{|l|}{ Sexual orientation* } \\
\hline Gay or bisexual & 3962 & $58(1.5)$ & $1.17(0.77$ to 1.80$)$ & 0.461 & & \\
\hline \multicolumn{7}{|c|}{ Number of partners $t$} \\
\hline$\leq 3$ & 2589 & $26(1.0)$ & 1 & Ref & 1 & Ref \\
\hline$>3$ & 4233 & $66(1.6)$ & 1.56 (0.99 to 2.47$)$ & 0.056 & 1.59 (1.01 to 2.51$)$ & 0.047 \\
\hline \multicolumn{7}{|c|}{ Urogenital chlamydia* } \\
\hline Negative & 5870 & $80(1.4)$ & 1 & Ref & & \\
\hline Positive & 295 & $2(0.7)$ & $0.49(0.12$ to 2.02$)$ & 0.326 & & \\
\hline
\end{tabular}

* Total values in these categories do not match total number due to missing data.

tOf any gender within the past 12 months.

TGD, transgender and gender diverse.

their medical history of sex with a TGD person were assigned a verified 'Yes' response. Those who had documented evidence in the electronic free-text field of not having had sex with a TGD person were assigned a verified 'No' response. If no evidence was recorded in a patients' medical history stating whether they had sex with a TGD person or not, they were excluded. Patients who responded 'No' were assumed to have given a verified response and therefore given a verified 'No' response. The proportion of verified responses was defined as those who had evidence of having sex with a TGD person after chart review divided by those who self-reported having sex with a TGD person on CASI. $\chi^{2}$ tests were used to examine the relationships between verified responses and patients' characteristics.

Univariable and multivariable logistic regression was performed to assess the association between the verified responses on having sex with a TGD person and patients' demographic characteristics (eg, age and country of birth), sexual behaviours (eg, number of partners in the past 12 months and sexual orientation) and urogenital chlamydia positivity. A sensitivity analysis was also performed to include both verified responses and unverified self-reported responses on having sex with a TGD person. First-void urine or urethral swab was taken for urogenital chlamydia screening using nucleic acid amplification test. Chlamydia is the only STI that both homosexuals and heterosexuals are routinely screened for currently, and therefore, it was the only STI included for analysis. Continuous variables such as age and number of sexual partners were categorised into groups at the median. Male and female patients were categorised into heterosexuals (sex with opposite-sex partners), gay/lesbian (sex with same-sex partners) or bisexual (sex with both same-sex and opposite-sex partners). Factors with a $\mathrm{p}<0.10$ in the univariable analysis were included in the multivariable analysis. All statistical analysis was carried out using Stata version 14 (Stata Corp, College Station, TX, USA).

\section{RESULTS}

There were 19176 male and female consultations during the study period; 8618 consultations were excluded because they were: not required to answer CASI $(n=8595)$, duplicate records $(n=19)$ or not a male or female patient $(n=4)$. Of the remaining 10558 consultations, 330 answered yes (3.1\%), 9796 $(92.8 \%)$ answered no, $152(1.4 \%)$ were unsure and $280(2.7 \%)$ declined to answer the question on having sex with a TGD. Of the 762 who answered yes, unsure or declined to answer, 458 $(60.1 \%)$ were excluded because they had no information in their medical record to indicate whether they had sex with a TDG person. Figure 1 details how patients were excluded from the final analysis. Following the selection criteria, there were 10100 consultations eligible for analysis.

Of the 10100 consultations included in the final data analysis, $6822(67.5 \%)$ were male and 3278 (32.5\%) were female. The age of the patients ranged from 16 to 82 years old, with a median age of 28 (interquartile range [IQR] 24-34). There were 4495 (44.5\%) patients born in Australia, 5231 (51.8\%) were born overseas and $374(3.7 \%)$ did not report their country of birth. The median number of self-reported sexual partners of any gender within the past 12 months was 4 (IQR 2-8). A minority of patients had injected drugs within the past 12 months $(1.4 \%)$ and few $(8.2 \%)$ reported currently working in the sex industry. Of the 6822 males, 3962 (58.1\%) were gay/bisexual, 2720 (39.9\%) were heterosexual males while the sexual orientation of $140(2.1 \%)$ men could not be determined. Of the 3278 females, $2852(87.0 \%)$ were heterosexual, 401 (12.2\%) were lesbian/ bisexual females with $25(0.8 \%)$ not reporting their sexuality. 
Table 3 Factors associated with having sex with a TGD person 3278 females attending the Melbourne Sexual Health Centre

\begin{tabular}{|c|c|c|c|c|c|c|}
\hline Factors & $\begin{array}{l}\text { Number of individuals, } \\
\mathrm{n}\end{array}$ & $\begin{array}{l}\text { Number of individuals } \\
\text { who had had sex with a } \\
\text { TGD person, } n(\%)\end{array}$ & $\begin{array}{l}\text { Crude OR } \\
(95 \% \mathrm{Cl})\end{array}$ & $P$ values & $\begin{array}{l}\text { Adjusted OR } \\
(95 \% \mathrm{Cl})\end{array}$ & $P$ values \\
\hline \multicolumn{7}{|l|}{ Age, years } \\
\hline $25-34$ & 1570 & $10(0.6)$ & 1.24 (0.45 to 3.42$)$ & 0.679 & & \\
\hline$\geq 35$ & 542 & $3(0.6)$ & 1.08 (0.27 to 4.32$)$ & 0.918 & & \\
\hline \multicolumn{7}{|l|}{ Country of birth* } \\
\hline \multicolumn{7}{|l|}{ Injecting drug use* } \\
\hline No & 3208 & $18(0.6)$ & 1 & Ref & 1 & Ref \\
\hline Yes & 20 & $1(5.0)$ & 9.33 (1.18 to 73.44$)$ & 0.034 & 2.73 (0.31 to 24.12$)$ & 0.365 \\
\hline \multicolumn{7}{|l|}{ Sexual orientation* } \\
\hline Heterosexual & 2852 & $6(0.2)$ & 1 & Ref & 1 & Ref \\
\hline \multicolumn{7}{|l|}{ Number of partners $\dagger$} \\
\hline$\leq 3$ & 1929 & $8(0.4)$ & 1 & Ref & & \\
\hline$>3$ & 1349 & $11(0.8)$ & 1.97 (0.79 to 4.92$)$ & 0.144 & & \\
\hline \multicolumn{7}{|l|}{ Urogenital chlamydia* } \\
\hline Negative & 2867 & $18(0.6)$ & 1 & Ref & & \\
\hline Positive & 196 & $0(0 \%)$ & - & - & & \\
\hline
\end{tabular}

${ }^{*}$ Total values in these categories do not match total number due to missing data.

tof any gender within the past 12 months.

\section{Verification of self-reporting sex with TGD}

Of the 151 people that self-reported 'Yes' on CASI to having sex with a TGD person and had evidence docunmented in patients' chart, there were 109 males (72.2\%) and 42 females (27.8\%). A total of 101 of these responses were verified on chart review, and this represented an overall $66.9 \%$ of patients. The proportion of verified responses was higher in males $(75.2 \%)$ than in females $(45.2 \%)(p<0.001)$. In males, there were no significant differences in the proportion of verified responses by demographic characteristics. The proportion of verified responses did not differ between heterosexual males $(73.7 \%)$ and gay/bisexual males $(76.1 \%)(p=0.785)$. In females, the proportion of verified responses reporting sex with a TGD person was higher among sex workers $(81.8 \%)$ compared with non-sex workers $(33.3 \%)$ $(\mathrm{p}=0.006)$. Although the proportion of verified responses among lesbian/bisexual females $(50.0 \%)$ was higher than heterosexual females $(37.5 \%)$, the difference was not statistically significant $(\mathrm{p}=0.429)$.

\section{The proportion of male and female having sex with a TGD person}

Based on the verified responses from the chart review, the overall proportion of males and females having sex with a TGD person was $1.0 \%(111 / 10100)$ (table 1). The proportion of males who had had sex with a TGD person in the past 12 months was higher than females $(1.3 \%$ (92/6822) versus $0.6 \%$ (19/3278); $\mathrm{p}=0.001$ ).

\section{Factors associated with having sex with a TGD person}

The multivariable logistic regression showed males aged 35 years and over compared with males aged 24 years and under had higher odds (adjusted OR (aOR) $=2.17,95 \%$ CI 1.14 to 4.14) of having sex with a TGD person after adjusting the number of male/female partners in the past 12 months (table 2). In males, sex with a TGD person was not associated with sexual orientation, injecting drug use, country of birth and sex work status. There was no association between urogenital chlamydia positivity and individuals who had sex with a TGD person in males $(\mathrm{OR}=0.49 ; 95 \% \mathrm{CI} 0.12$ to 2.02$)$. In females, lesbian/bisexual females had higher odds $(\mathrm{aOR}=13.69,95 \% \mathrm{CI} 5.06$ to 37.02$)$ of having sex with a TGD person than heterosexual females after adjusting other confounding factors including country of birth, injecting drug use and sex work (table 3). Unlike males, age was not associated with having sex with a TGD person in females. There was no difference in urogenital chlamydia positivity between females who had sex with a TGD person $(0 \%)$ and those who did not have sex with a TGD person $(0.6 \%)$ $(p=0.625$, Fisher's exact test). Among the 101 verified responses on having sex with a TGD, only one male was newly diagnosed with HIV and he was an MSM.

A sensitivity analysis was also performed to include 179 individuals (119 males and 60 females) who self-reported having sex with a TGD person but no documented evidence recorded in patients' chart review (figure 1). The inclusion of these individuals identified some additional significant factors associated with having sex with a TGD person in both males (online table S1) and females (online table S2); however, both analyses consistently showed that urogenital chlamydia positivity was not associated with having sex with a TGD person in both males and females.

\section{DISCUSSION}

In this evaluation of a novel question asking about TGD partners using CASI, 151 (1\%) individuals self-reported having a TGD partner which, in $66.9 \%$ of cases, was verified after reviewing their medical records and about one-third of the 
responses could not be verified. This study shows that males $(1.3 \%)$ were more likely to have had sex with a TGD person than females $(0.6 \%)$ in the past 12 months. Older males were also more likely to have sex with a TGD person than younger males, but no age pattern was observed in females. Sexual orientation appeared to have no association with sex with a TGD person in males. In contrast, lesbian/bisexual females were more likely to have sex with a TGD person than heterosexual females. The verified response rate was higher in males (75.2\%) than females (45.2\%). Female who were sex workers $(81.8 \%)$ had a higher verified response rate compared with females who were not sex workers (33.3\%). There was no association between chlamydia positivity and individuals who had sex with a TGD person.

Our study has several limitations. Firstly, it is not a current routine clinical practice to verify and document when the patients self-reported having sex with a TGD person via CASI, so in over half of individuals their self-reported responses to 'yes', 'declined to answer' or 'unsure' could not be verified. This explains why $60.1 \%$ (456/762) of consultations were reviewed and then excluded which may have provided a source of bias. Secondly, the data were collected from a single sexual health clinic in Melbourne, which means the data are unlikely to represent a broader population such as Australia or other countries. This is because the number of TGD people or people having sex with a TGD person in a population can be influenced by social norms that vary from place to place. ${ }^{12}$ Thirdly, we were only able to assess urogenital chlamydia as an STI measure but not other STIs including HIV. This is because HIV and other STI such as syphilis and gonorrhoea were not routinely screened among heterosexuals as per the Australian STI testing guidelines during the study period. Fourthly, 9796 individuals self-reported 'no' to the sex with TGD person, and these responses were not verified via chart review. The time this would have taken made this impractical, and it was likely this would not be verified during the consultations and documented in the patients' record. Finally, we did not formally assess the patients understanding of this question in CASI but appreciate that undertaking some qualitative research would be of substantial value.

This study has shown that the recent introduction of the question on sex with a TGD person via CASI has been valuable, particularly in males, as it has identified of those having sex with TGD people. In females, the response on CASI should be interpreted with caution, particularly among females who are not sex workers, as only $33.3 \%$ of the responses were verified. We have also been able to examine some of the factors that may or may not make a person more likely to have had sex with a TGD person. Again, this should aid clinicians in identifying those who are having sex with TGD people.

It was apparent while conducting the chart review that a significant number of patients confused the term transgender with bisexual or homosexual. However, given our current data, it is not possible to precisely quantify the number of patients who misunderstood the TGD question. We feel this would be of use to know, particularly in assessing people's understanding of the terms 'transgender' and 'gender diverse'. We believe a survey or interviews may be required to assess the discordance between self-reported and verified responses to the TGD question to further understand the understanding and awareness of this terminology in the general population. Although some patients indicated they were confused with the terminology, no patients formally complained and reported any discomfort about the question on TGD for the duration of the study. The service receives several hundred regular written feedback from patients each year.

There are very limited studies evaluating the risk of HIV/ STI among individuals who had sex with a TGD person. In our study, we did not find an association between chlamydia positivity and individuals who had sex with a TGD. A study in San Francisco analysed data from a large database with 13754 individuals and reported that there was no difference in HIV positivity between individuals who had sex with a TGD and those who did not. ${ }^{13}$ McNulty and Bourne ${ }^{14}$ have identified that there are very limited studies on STI prevalence among TGD population in their review; however, they reported that the STI prevalence among TGD in Australia is generally lower compared with TGD in other countries, particularly in resource-poor settings.

In addition, TGD population is also facing several other health issues such as mental health, violence and abuse. ${ }^{15}$ Stigma and social discrimination may create a social barrier for the TGD population in accessing and seeking healthcare. ${ }^{16} 17$ Furthermore, lack of knowledge for the needs of TGD among healthcare professionals has also been identified as a major barrier. ${ }^{18} 19$ Adequate and updated training for healthcare professionals on clinical management and care for TGD population is urgently needed. ${ }^{20}$

Among the patients attending at our sexual health centre, we could verify most $(66.9 \%)$ of the patients who self-reported having sex with a TGD person. This shows that the introduction of the TGD question on CASI in addition to be inclusive has also proven to be useful in determining those who had sex with TGD people. Accuracy could be further improved by including a definition of 'TGD' on CASI. We contend this question should continue to be asked to all male and female patients in order to provide better clinical management and care for the individuals. Although there was no difference in urogenital chlamydia between individuals who had sex with a TGD person and those who did not, more research is required to understand the sexual practices with TGD person in order to understand the risk of HIV and other STIs such as syphilis and gonorrhoea, which may have a different mode of transmission compared to chlamydia. This information would be important for clinical management. It is also possible that TGD persons can also have sex with another TGD, and more specialised clinical care should be applied to the TGD population. Due to the complexity of the questions among TGD population, more research and consultations with the TGD community may be required to determine the most appropriate and accurate questions for TGD.

\section{Key messages}

- Having sex with a transgender or gender diverse (TGD) person is not currently included as part of a standard sexual history in Australian and International guidelines.

- A question on sex with a TGD person was asked to all patients attending a sexual health centre in Melbourne, and most of the responses (66.9\%) were verified and patients did not feel any discomfort.

- Males (1.3\%) were more likely to have sex with a TGD person than females $(0.6 \%)$.

- There was no association between urogenital chlamydia positivity and individuals who had sex with a TGD person in both males and females. 


\section{Handling editor Jonathan Ross}

Acknowledgements We acknowledge Afrizal Afrizal for his assistance with data extraction and Jun Kit Sze implemented this question on CASI.

Contributors CKF and EPFC contributed to the conception of this study. EFC conducted literature review, performed data analysis and wrote the first draft of the manuscript. EPFC assisted with statistical analysis. All authors were involved in data interpretation, revised the manuscript critically for important intellectual content and approved the final version.

Funding EPFC is supported by the National Health and Medical Research Council (NHMRC) Early Career Fellowship (1091226).

Competing interests None declared.

Patient consent Not required.

Ethics approval Ethical approval was obtained from the Alfred Hospital Ethics Committee, Melbourne, Australia (project number 539/17).

Provenance and peer review Not commissioned; externally peer reviewed.

(c) Article author(s) (or their employer(s) unless otherwise stated in the text of the article) 2019. All rights reserved. No commercial use is permitted unless otherwise expressly granted.

\section{REFERENCES}

1 Ainsworth C. Sex redefined. Nature 2015:518:288-91.

2 Judith L. Beyond the Binaries: Depolarizing the Categories of Sex, Sexuality, and Gender. Sociological Inquiry 1996;6660:143.

3 Lo S, Horton R. Transgender health: an opportunity for global health equity. Lancet 2016;388:316-8.

4 Reisner SL, Poteat T, Keatley J, et al. Global health burden and needs of transgender populations: a review. Lancet 2016;388:412-36.

5 Baral SD, Poteat T, Strömdahl S, et al. Worldwide burden of HIV in transgender women: a systematic review and meta-analysis. Lancet Infect Dis 2013;13:214-22.

6 Bauer GR, Scheim Al. Sampling bias in transgender studies. Lancet Infect Dis 2013;13:832.

7 Tideman R, Pitts M, Fairley CK. Use of the Delphi sorting technique to establish a core sexual history. Int I STD AIDS 2006;17:170-2.
8 Brook G, Bacon L, Evans C, et al. 2013 UK national guideline for consultations requiring sexual history taking. Clinical Effectiveness Group British Association for Sexual Health and HIV. Int J STD AIDS 2014;25:391-404.

9 Grosz AM, Gutierrez D, Lui AA, et al. A Student-Led Introduction to Lesbian, Gay, Bisexual, and Transgender Health for First-Year Medical Students. Fam Med 2017;49:52-6.

10 Gamagedara N, Dobinson S, Cummings R, et al. An evaluation of an express testing service for sexually transmissible infections in low-risk clients without complications. Sex Health 2014;11:37-41.

11 Chow EPF, Fortune R, Dobinson $S$, et al. Evaluation of the Implementation of a New Nurse-Led Express "Test-And-Go" Human Immunodeficiency Virus/Sexually Transmitted Infection Testing Service for Men Who Have Sex With Men at a Sexual Health Center in Melbourne, Australia. Sex Transm Dis 2018;45:429-34.

12 Atkinson SR, Russell D. Gender dysphoria. Aust Fam Physician 2015;44:792-6.

13 Wilson EC, Chen YH, Raad N, et al. Who are the sexual partners of transgender individuals? Differences in demographic characteristics and risk behaviours of San Francisco HIV testing clients with transgender sexual partners compared with overall testers. Sex Health 2014;11(4):319-23.

14 McNulty A, Bourne C. Transgender HIV and sexually transmissible infections. Sex Health 2017;14:451-5.

15 Veale JF, Peter T, Travers R, Vj F, Tracey P, Robb T, et al. Enacted Stigma, Mental Health, and Protective Factors Among Transgender Youth in Canada. Transgend Health 2017:2:207-16.

16 White Hughto JM, Reisner SL, Pachankis JE. Transgender stigma and health: A critical review of stigma determinants, mechanisms, and interventions. Soc Sci Med 2015;147:222-31.

17 Dowshen N, Lee S, Franklin J, Nadia D, Susan L, Joshua F, et al. Access to Medical and Mental Health Services Across the HIV Care Continuum Among Young Transgender Women: A Qualitative Study. Transgend Health 2017;2:81-90.

18 Edmiston EK, Donald CA, Sattler AR, et al. Opportunities and Gaps in Primary Care Preventative Health Services for Transgender Patients: A Systemic Review. Transgend Health 2016:1:216-30.

19 Kevan W, Rebecca W. Supporting trans people in clinical practice. Trends in Urology \& Men's Health 2016;713:9.

20 Wylie K, Knudson G, Khan SI, et al. Serving transgender people: clinical care considerations and service delivery models in transgender health. Lancet 2016;388:401-11. 